Proceedings of the Creative Construction Conference (2018)

Edited by: Miroslaw J. Skibniewski \& Miklos Hajdu

DOI 10.3311/CCC2018-002

Creative Construction Conference 2018, CCC 2018, 30 June - 3 July 2018, Ljubljana, Slovenia

\title{
Are computers agents? Considering the implication of classifying computers as occupants on energy consumption and proximity-as- utility equipment scheduling
}

\author{
Stephen Oliver, Dr. Farzad Pour Rahimian
}

Univeristy of Strathclyde, Glasgow, UK

\begin{abstract}
Non-ancillary equipment makes significant contribution to building energy demand, consuming as much as half of total energy consumption and three quarters of consumption during inoccupancy. Current proximity-as-utility equipment scheduling does not reflect physical or social complexities of mediating equipment, failing to suitably represent equipment and seemingly incompatible with inoccupancy scheduling. This paper draws comparison between computers and conventional occupants attempting to identify the extent which it is applicable to energy modelling. It concludes with the concession of its lack of relevance in inoccupancy equipment scheduling, though inherently convenient and suggests partial decoupling of agents and non-ancillary equipment during occupancy.
\end{abstract}

Proximity-as-utility is herein defined as equipment scheduling defining utility as a Boolean-state power density necessitating the presence of a proximal agent, accommodating short periods where equipment and agent do not cohabit a discrete space.

(C) 2018 The Authors. Published by Diamond Congress Ltd., Budapest University of Technology and Economics Peer-review under responsibility of the scientific committee of the Creative Construction Conference 2018.

Building simulation; occupant ontology; virtual actors; moral agents

\section{Introduction}

Ostensibly, from the planets in a solar system to people in buildings everything is an occupant and ecosystem to some extent. Each are nested and/or interacting, non-stationary systems with their own diverse occupants. Systems are varyingly persistent and prone to traversal through parent systems. Their relationships with other systems and processes can be mutually exclusive or intricately linked, but even those which are seemingly distal can have a persistent knock-on effect on both physical and optimum state definitions.

The implied feature is being human is not a prerequisite of occupant status, something which is becoming more prevalent in the literature. Furniture for example, in the form of spatially-represented internal thermal masses [1] which $\mathrm{Li}, \mathrm{Xu}$ [2] note as contrasting with existing model assumption of an isothermal stability between furnishings and the space. Furthering this, Johra and Heiselberg [3] discuss the validated relationship between comfort and

Corresponding author: Stephen Oliver; email: stephen.oliver@strath.ac.uk 
furnishing, specifically humidity buffering, and indicate the need for further research into the complex effects of furniture.

Previous building physics research discussed what could be considered the impact of equipment personalities on energy demand with [4] identifying an established case for equipment similar to the theme of this article proclaiming "simple workloads" fail to reflect the complexity and variety of user or equipment behaviours. Jones et al effectively discuss the hardware personalities and identify idiosyncrasies in the form of mixed focus software activity, thought they do not consider the relationship between the agent and mediator, the computer. Social science has investigated the effect of perceived humanity in electronic devices on people's behaviours and perceptions of the world around them [5] [6] which is supported by computer ethics literature Johnson and Powers [7],Nonetheless, scheduling for equipment is still largely proximity-as-utility, aggregated power densities.

Current scheduling where consumption is a feature of occupant presence rather than the equipment itself, does not appear to sufficiently encompass the intricacies of non-ancillary equipment and inherently ill-prepared to support equipment scheduling for inoccupancy. However, the extent in which a change to the existing model is required, especially in terms of consumption during inoccupancy, is unclear. This paper discusses non-ancillary equipment scheduling and physical/social personalities using computers, the most significant contributor to plug load and diverse form of non-ancillary equipment, to assess the proposal.

\subsection{Structure}

Literature from peer-reviewed journals, primarily sourced from Science Direct, were identified which demonstrate the significance of computer energy consumption during occupancy and inoccupancy. Existing research on similar themes of inanimate agents and computer personalities from building servicing and social science literature were used to draw comparison between computers and agents. Observations were aggregated into general statements equipment scheduling to show relevance or lack thereof for classifying computers as agents during occupancy and inoccupancy, separately.

\subsection{Precedence}

Discussion on disaggregation of occupant-attributed disturbances has received increasing attention over the last few decades. The suggestion that some equipment are closer to being occupants than equipment in the conventional sense has in principle been covered previously in activity detection $[4,8]$ where appliance-level consumption was considered significant to understanding user behaviours. Jones, Wei [4] discussing system processes and energy consumption implicitly suggest the impact of idiosyncratic personalities of the machines themselves. Personality seems further supported by Joy E, Suart A [9] who note agents are not exclusive to a single computing devices or locations and Gunay, O'Brien [10] who identify significant differences in the energy conscious behaviours of respondents when using differing computer types. Information technology and social science literature makes a contrasting but complementary case for treating computers people and how this naturally manifests in one-way [11] and two-way [6] relationships between people and electronic devices. Similarly, computer ethics research does not consider treating computers as "surrogate" agents a novel idea [7].

\subsection{Significance}

Equipment operation is often considered a passive occupant behaviour, one which does not interfere directly with ancillary services, yet some forms of equipment are arguably better thought of as agents, closer to the definition of conventional occupants than equipment. It seems like an oversight in taxonomy to simplify it to a passive disturbance from the occupant when plug loads alone contribute $12 \%$ to $50 \%$ of energy use in offices [10, 12], and up to and beyond $50 \%$ of commercial building energy is consumed outwith operational hours [13]. Gunay, O'Brien [10] observed up to $75 \%$ of plug load energy use being attributed to out of hours consumption in offices, $70 \%$ of plug loads associated with personal computers with around $60 \%$ private occupants using desktop computers, only $50 \%$ of which report switching computers off at end of day. However, the latter observation is contrary to those of Wang and Ding [14] who observed $90 \%$ of users switching off during predictable periods of absence They also notice the appearance of more energy-awareness from laptop users with $25 \%$ of computer users reporting never turning off in contrast to only $5 \%$ of laptop users and $10 \%$ laptop users shutting down during intervals. Jones, Wei 
[4] monitored energy consumption to extrapolate and potential energy savings from computer users suggesting global computer energy consumption could be reduced by $17 \mathrm{TWh}$ with lower thresholds for standby mode, and 230 $-68 \mathrm{GWh}$ through reduction LED backlight intensity, per annum. Though they acknowledge their sample size is too small to make definitive conclusions. An interesting secondary observation was the persistence of web-based advertisements in browser and/or tabs which were not in focus increasing the number of active processes suggesting something as simple an ad-blocking tool could reduce energy consumption. Ethical quandary of ad-blocking aside, these show not only is equipment-level user behaviour monitoring desirable, but the personality of the system itself is worth considering. The problem extends to residential buildings where it is expected equipment energy consumption will tripled by 2030 from 2009 levels [4]. Zhang and Bai [15]'s study of consumer goods ownership in Shangdong identified a 600\% increase in computer ownership per hundred urban households between 2005 and 2013 indicating a drastic surge in electronic energy consumers.

Given these observations and case study observation of high utilisation variance [14] it seems evident that nonancillary equipment are not entirely compatible with proximity-as-utility scheduling as is available with the decoupled scheduling used in DSMs or presence-as-utility in lower-precision models, the latter assuming any presence is merits utility. That is, during occupancy the activities and behaviours of conventional occupants and computers aren't entirely dependent and it's not only the conventional occupants' disturbance to the building ecosystem which isn't steady-state.

\subsection{Computer and equipment in buildings}

Computers are distinct in building energy contexts from other equipment in several ways but the most significant is their position between ancillary and non-ancillary stationary equipment where the latter can be managed by smart controllable load (SCL) automation [16] and the former's operational schedule is immutable, refrigerators, routers or servers for example. Computers fall under neither category; user inactivity is not an indication of activity or inactivity of the device, but they are mutable and increasingly portable. Additionally, computers and SCLs can have human-mediated relationships both of which can be supported by supervised or unsupervised optimisation $[16,17]$. Similarly, computers can dictate conventional occupants' actions whether actively through delivering information or passively through processing affecting occupants' activities within a building, although their mobility is dependent on the conventional occupant, the occupant's behaviours can be guided be the device.

Herein lies the problem, non-ancillary equipment, particularly computers' consumption mutability is situational and a product of the relationship between the conventional occupant, the computer and the collective energy culture of the occupants. In contrast, services follow rules and adapt, and ancillary equipment consumption is immutable. Even disregarding equipment personalities, existing equipment scheduling not reflect divergence between ancillary and non-ancillary equipment personalities.

\section{Computers as moral and social entities}

Laptops are arguably similar to young children to many building service configurations' interpretations of an occupant in their ecosystem, differing primarily in flexibility of shared characteristics. Both passively contribute heat to the parent ecosystem at activity and environment-dependent rates, depend on adult occupants for mobility, not meaningfully able to interact with the parent ecosystem without adult guidance and appear to stochastically exhibit signs of sentience in varyingly significant and consistent forms. In the case of the latter, people mindlessly treat computers as humans (ethopeia) [6] which they do fairly liberally [18] and adults pretend children have selfawareness beyond the child's age, suggesting similarities in status as social actors and to an extent, the appearance of self-efficacy. In contrast, children do not generate light pollution and computers do not directly emit pollutants affecting humidity or air quality and are entirely reliant on conventional occupants making a distinction between child and laptop readily available to sensor-supported conditioning. Nonetheless, they interact with the environment beyond thermal disturbance through interaction with other inhabitants and immaterial environmental pollution, appearing to favour a less simplistic object model for equipment at least for some non-ancillary devices. Laptops in particular have one characteristic exclusive to portable devices which has been shown to complicate power density reporting [10], they transfer energy and information between adjacent and/or consumed and distal ecosystems.

Looking at it from computer ethics perspective perhaps best alludes to why social personality is important. Discussing surrogate agency Johnson and Powers [7] notes the presence of spyware or bots as evidence the idea 
computers are agents, in ethical contexts, is not novel. The statement supports the observation of Jones, Wei [4] on the effect of software on a computer's personality (activity) but its primary relevance to this discussion is defining computers as at minimum, surrogate agents with "second-order interests" akin to the relationship between a lawyer and client. In an earlier article, Johnson [19]Johnson [19] explains "... as with human behavior, when computer systems behave, their behavior has effects on other parts of the embodied world". This is a critical feature of the proposed reclassification, the effect of their behaviour (personality) does not exclusively reside within the mind of the user, which complements Jones et al and Wang's energy and social personalities. However, Johnson later highlights computer systems' intentionality is "inert" or "latent" without the intentionality of the user which, in terms of this article, seems to nullify any significant value in considering computers as agents during inoccupancy.

People assign humanity to computers whilst fully aware of the absurd attribution, nonetheless, they form relationships which are sensitive to the computer's ability to manage its second-order interests, similar to the way an office worker's employment is dependent on their second-order interest, keeping management satisfied. However, a computer's agency is dependent on the intentionality of the user and therefore is removed when the user and latent intentionality expires. This seems suggests computers are agents during occupancy but not inoccupancy.

\section{Discussion}

Computers share similarities with conventional occupants not quite fitting into any category of equipment, exhibiting similar physical and social characteristics of occupants and developing relationships with the user and world around them. They interact distinctly from other forms of equipment and contribute a significant portion of building energy consumption which was shown to have justification for increasing equipment-occupant interactions modelling precision to software and hardware level. Their contributions to the ecosystem have similarities to young children having dependent mobility and creating a mixture of material and immaterial pollutants causing environmental and social events, arguably with a higher chance of passing the Turing test. That is, the information they transmit to the environment has more utility and capacity to change the environment with greater persistence. Their environment disturbances are also activity-dependent in a manner which is neither Boolean nor reliant on physical or SCL device intervention. Finally, their behaviour is learned from peers though dependent on user intentionality and they can transfer electrical potential and information between environments which, in the case of information does not require spatial proximity.

However, although computers and occupant function are co-dependent in office settings conventional occupants in isolation are independent from computers. Therefore, computers are subordinate to the conventional occupants meaning the stochastic nature of their environmental and social impact do not persist during inoccupancy and effectively are akin to an ancillary device.

The underlying principle of considering computers as more than static sources of disruption and consumption as individual entities sharing characteristics of conventional occupants if only during occupancy is significant and validated by existing research. In terms of inoccupancy it appears the proposal has some merit for filling the absent consumption void of existing proximity-as-utility scheduling however, with computers robbed of agency during inoccupancy there is no necessity for any complex behavioural modelling beyond determining appropriate loads, power state probabilities and durations of inoccupancy.

The transition between agent and equipment status and the resulting Boolean-state power density is difficult to quantify. Jones et al observed a significant number of computer users not powering off yet not so many with laptops. This could be situational or cultural but equally could just be the ergonomic benefits from linking screen hinge angle and power state. Energy-conscious behaviours stem from education and sociocultural pressures which suggests when devoid of personality, the consumption associated with non-ancillary equipment during unoccupied periods is primarily a function of social exposure or the conventional occupant's desire for exposure. If this is the case, then non-ancillary equipment inoccupancy scheduling may be considered culture-as-utility or identity-as-utility depending on the desired granularity.

Jones et al have laid the foundation for the latter with their software, but cultural weighing is beyond the scope of this article or the referenced literature. The authors would recommend future research into cultural consumption and sensitivity analysis for occupant wellbeing assessment. 


\section{Conclusion}

High consumption during inoccupancy, total plug load contribution and supporting device monitoring literature highlight the necessity to re-evaluate the what we consider to be an occupant in building energy simulations or predictions. Treating non-ancillary equipment as subordinate agents rather than simply power densities during occupancy appears to be a necessary feature for researchers investigating energy conservation measures, the performance gap or real-time monitoring but their status changes to a culture-sensitive disturbance during inoccupancy.

This paper proposed non-ancillary equipment, primarily computers, should be considered as agents rather than Boolean-state power densities or features of the conventional occupant. Since the computer's agency is dependent on the presence of a conventional occupant they are subordinate and are effectively fall back to the conventional definition of equipment during inoccupancy. To this end the author maintains the relevance of the premise of computers as agents during occupancy for energy and wellbeing but acknowledges there is little value in considering them as agents during inoccupancy.

\section{Acknowledgements}

This paper is the product of the support and tolerance of Maureen Eisbrenner of arbnco and Strathclyde University's architecture department for which I am very grateful. A secondary acknowledgement of Michael Reilly BEng, building services engineering consultant for his invaluable feedback from the perspective of a building services engineer.

\section{References}

1. Raftery, P., et al., Effects of furniture and contents on peak cooling load. Energy and Buildings, 2014. 85: p. 445-457.

2. Li, W., et al., A new method for calculating the thermal effects of irregular internal mass in buildings under demand response. Energy and Buildings, 2016. 130: p. 761-772.

3. Johra, H. and P. Heiselberg, Influence of internal thermal mass on the indoor thermal dynamics and integration of phase change materials in furniture for building energy storage: A review. Renewable and Sustainable Energy Reviews, 2017. 69: p. 19-32.

4. Jones, M.E., B.W.Y. Wei, and D.L. Hung, Laptop energy-saving opportunities based on user behaviors. Energy Efficiency, 2013. 6(2): p. 425-431.

5. Cliffard, N., J. Steuer, and E.R. Tauber, Computers are social actors. CHI '94 Proceedings of the SIGCHI Conference on Human Factors in Computing Systems, 1994(72-78).

6. Wang, W., Smartphones as Social Actors? Social dispositional factors in assessing anthropomorphism. Computers in Human Behavior, 2017. 68: p. 334-344.

7. Johnson, D.G. and T.M. Powers, Computers as surrogate agents, in Information Technology and Moral Philosophy, M.J. van den Joven and J. Weckert, Editors. 2008, Cambridge University Press. p. 251.

8. Ahmadi-Karvigh, S., et al., Real-time activity recognition for energy efficiency in buildings. Applied Energy, 2018. 211: p. 146-160.

9. Joy E, P., et al., A SURVEY OF COMPUTER POWER MODES USAGE IN A UNIVERSITY POPULATION. California Energy Commission, 2014: p. 29-39.

10. Gunay, H.B., et al., Modeling plug-in equipment load patterns in private office spaces. Energy and Buildings, 2016. 121: p. 234-249.

11. Klimmt, C., T. Hartmann, and H. Schramm, Parasocial interactions and relationships. 2006. 291-313.

12. Gandhi, P. and G.S. Brager, Commercial office plug load energy consumption trends and the role of occupant behavior. Energy and Buildings, 2016. 125: p. 1-8.

13. Zhao, J., et al., Occupant behavior and schedule modeling for building energy simulation through office appliance power consumption data mining. Energy and Buildings, 2014. 82: p. 341-355.

14. Wang, Z. and Y. Ding, An occupant-based energy consumption prediction model for office equipment. Energy and Buildings, 2015. 109: p. 12-22.

15. Zhang, M. and C. Bai, Exploring the influencing factors and decoupling state of residential energy consumption in Shandong. Journal of Cleaner Production. 
Stephen Oliver, Dr Farzad Pour Rahimian / Proceedings of the Creative Construction Conference (2018) 000-000

16. Martinez-Pabon, M., T. Eveleigh, and B. Tanju, Optimizing residential energy management using an autonomous scheduler system. Expert Systems with Applications, 2018. 96: p. 373-387.

17. Robillart, M., et al., Model reduction and model predictive control of energy-efficient buildings for electrical heating load shifting. Journal of Process Control, 2018.

18. Lee, K. and C. Nass, Designing Social Presence of Social Actors in Human Computer Interaction, Computer Human Interaction 2003. Vol. 5. 2003. 289-296.

19. Johnson, D.G., Computer systems: Moral entities but not moral agents`. Ethics and Information Technology, 2006. 8: p. 195-204. 